\title{
A study for semi-automatic diagnosis support of strokes from 2D MRI/FLAIR sequences
}

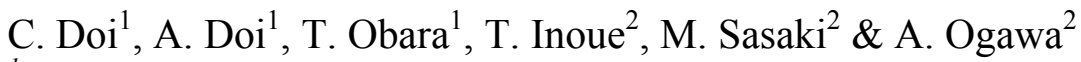 \\ ${ }^{1}$ Iwate Prefectural University, Japan \\ ${ }^{2}$ Iwate Medical University, Japan
}

\begin{abstract}
The MRI/FLAIR sequence is being used for the diagnosis of strokes. The bleeding area shows a high intensity in most cases. However, a doctor must check each image which can take a lot of time. We found that in this process a skilled doctor typically checks the gap area (internal cavity) of the brain to give a diagnosis. This fact is confirmed by a questionnaire of several neuro surgeons. In order to extract the gap area, we utilize a region growing method and a 2D deformable model. We also improve the preciseness by eliminating high intensity areas near the bulb of the brain. Finally, we calculate the degree of riskfactor by counting pixels with high intensity in the gap area. From our experience, we found that the histogram of strokes had a similar pattern, and it could be used for diagnosis of strokes. Finally, we visualize the distribution of the bleeding area of the stroke, and evaluate the results in several ways.

Keywords: diagnosis support, stroke, MRI, region growing method, image processing.
\end{abstract}

\section{Introduction}

Every year a very large number of people suffer from strokes. It is necessary for stroke victims to take an urgent treatment, because the death rate is very high, and the after-effects, such as paralysis of one side of the body, are common. Both $\mathrm{CT}$ and MRI are being used for the diagnosis of strokes. A bleeding area in CT and MRI shows high intensity in most cases. However, small cerebral hemorrhage often leads to misdiagnosis, since the area of bleeding is too small, and the intensity in the area is low. This often happens in the emergency hospital where a neuro surgeon is not present. Moreover, a doctor also must check each 
image which can take a significant amount of time, since recent CT and MRI can be scanned in a short period, and they generate many image slices.

In order to overcome the problems, we propose a semi-automatic diagnosis method for strokes, which estimate the risk-factor of stroke automatically from two-dimensional MR image. Our approach judges for every two-dimensional MR/FLAIR sequence (FLuid-Attenuated Inversion Recovery), and it indicates a risk-factor and the bleeding area on a monitor. The FLAIR pulse sequence is widely used in the diagnosis of diseases of the brain because of spurious high signals in blood flow and cerebrospinal fluid (CSF). This newer MR techniques are available on advanced MR systems. In this paper, we assumed that $\mathrm{MRI} /$ FLAIR pulse sequence is used in diagnosis of strokes.

We found that skilled neuro surgeons typically checked the gap area of the brain to give a diagnosis. This fact is confirmed by a questionnaire of several neuro surgeons. To mimic the diagnosis process of a skilled doctor, we derive the following steps:

1) Obtain MRI/FLAIR sequences of the patient's brain

2) Segment the brain, blood vessels, wrinkle area, etc.

3) Extract the gap area of the brain from MRI

4) Analyze the pixels in the gap area to estimate whether the patient has a stroke or not.

In order to extract the gap area, we utilize a region growing method [1-4] and a 2D deformable model [5-7]. Finally, we calculate a risk-factor by counting pixels with high intensities in the gap area. By calculating each slice of MRI/FLAIR sequences, a doctor can check the slices of high risk-factor values easily (Fig. 1).

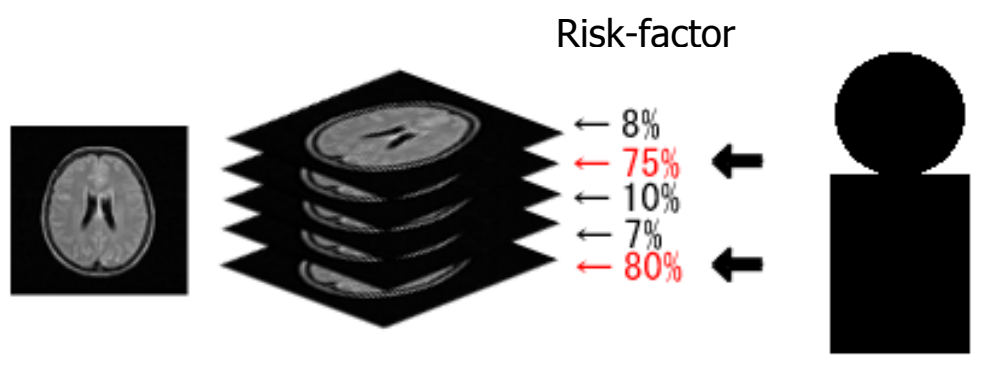

Figure 1: Concept of our diagnosis support system.

\section{Proposed method}

When bleeding due to the brain internal hemorrhage is a small quantity, an experienced neuro surgeon pays attention to the gap area of the brain in each image slice, and find the bleeding, and diagnoses the patient. In order to mimic the diagnosis process of a skilled doctor, we derive the following steps:

1) The MR slice images (two-dimensional images) of the patient's brain are acquired. 
2) The region of the patient's cerebrum is extracted by using a region growing (RG) method. The initial seed point and the merging criteria are specified interactively.

3) By taking the difference between the region of the cerebrum and the convex area of the extracted cerebrum, the gap area of the brain and the other area with different intensity values are extracted.

4) The part of the blood vessel is removed from the gap area of the brain, because the area of the blood vessels has high intensity values.

5) By checking the pixels in the area that extracted in Step 4, we estimate whether the patient has a stroke or not. We also display the bleeding area on monitor.

Fig. 2 shows an overview of our proposed method, and we utilize an original MRI/FLAIR sequence, and the data format is DICOM (Digital Imaging and Communications in Medicine). By using this figure, we explain the overview of our approach. A region growing (RG) method is one of image segmentation techniques, and it starts from some pixels (seeds) representing distinct image regions and to grow them, until they cover the entire image. The constraints are specified either as a threshold range (a minimum and maximum pixel value) or as a multiple of the standard deviation of the region pixel values. Images segmented by region growing methods often contain either too many regions (under-growing) or too few regions (over-growing) as a result of non-optimal parameter setting.

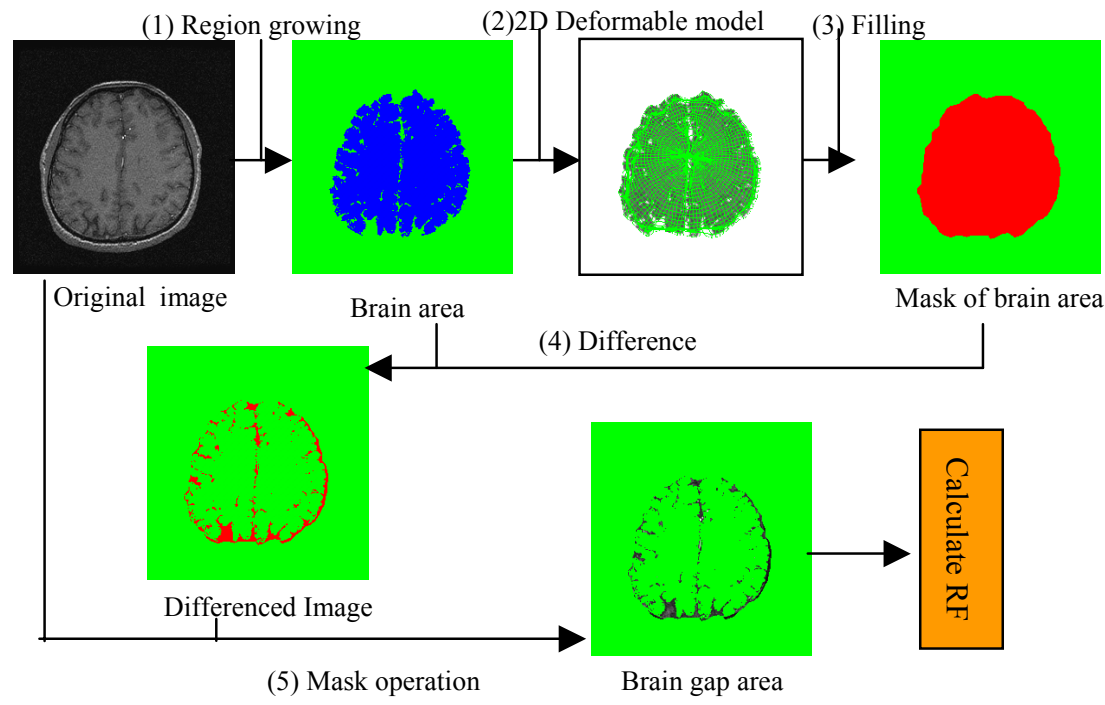

Figure 2: $\quad$ Flowchart of our diagnosis support system.

To improve classification results, we use an edge-enhanced filter, such as Sobel filter. Fig. 2 shows that the blue area is a segmentation result of cerebrum with similar pixel intensity by using RG method. We select the seed point and parameters of RG method interactively. In order to get the gap area of the brain, 
at first a convex hull that encloses the extracted brain (red area) is calculated by using 2D deformational model, which we called "2D Active Net". 2D Active Net consists of a circle-shaped geometry model as an initial form, and it deforms the shape by iteration of the computation based on the energy minimization theory. Next, we define a risk-factor of stroke, $\mathrm{T}$ by the following equation.

$$
\mathrm{T}=\mathrm{Nc} / \mathrm{Nt}
$$

Here, $\mathrm{Nc}$ is a number of the pixel higher than a threshold $\mathrm{C}, \mathrm{Nt}$ is a total number of pixel inside the difference mask image. The threshold $\mathrm{C}$ is specified by neuro surgeon interactively or automatically by our system.

Fig. 3 shows one of the results of our method. The left image is the original MRI/FLAIR. This MRI/FLAIR sequence was taken on the next day after the patient got ill. The centred image shows the estimated bleeding areas of first stage of our method. The right image shows the wrong estimated regions (enclosed by green lines by neuro surgeon). We had a questionnaire to three neuro surgeons for the results. Fig. 4 shows the histogram of the slices in the estimated bleeding regions.
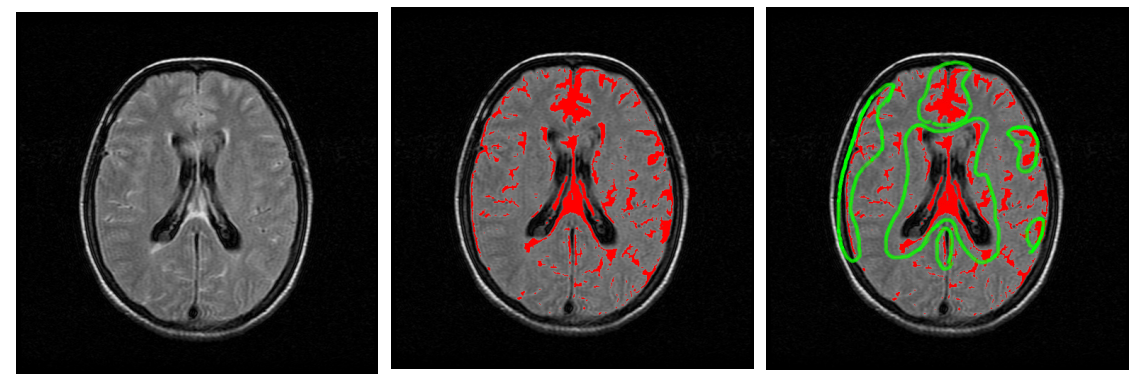

Figure 3: Original image, Estimated bleeding area, and Wrong estimated area (green lines).

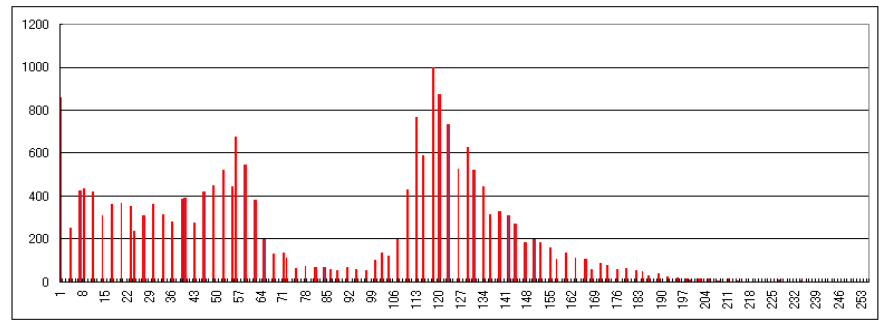

Figure 4: $\quad$ Histogram of the gap area.

The histogram of the patient with stroke shows that it has a ridge and two peaks. The right side of peak shows the bleeding area of the patients. In this case, the neuro surgeon specifies the threshold $\mathrm{C}$ as 90 by using the histogram. As a 
result, the risk-factor of stroke in Fig. 3 is 0.489476 (the number of pixels over 90: 10232, the total number of pixels: 20904).

As the reason of wrong estimation, we found that MRI/FLAIR has the feature that areas near bulb of brain have high intensity, and we set the threshold $\mathrm{C}$ at a little lower position.

Therefore, we apply the two improvements in our diagnosis support system.

(1) Setting a threshold $\mathrm{C}$ at the position of maximum value of the histogram.

(2) Elimination of high intensity area of bulb of brain.

We also change the manner of specifying a threshold $\mathrm{C}$. We decide to select the maximum value in right peak of histogram. In Fig. 4, the maximum threshold $\mathrm{C}$ becomes 119. It is possible to specify it automatically in diagnosis support system. We also extract the brain bulb, and expand the area, and eliminate the area from bleeding area.

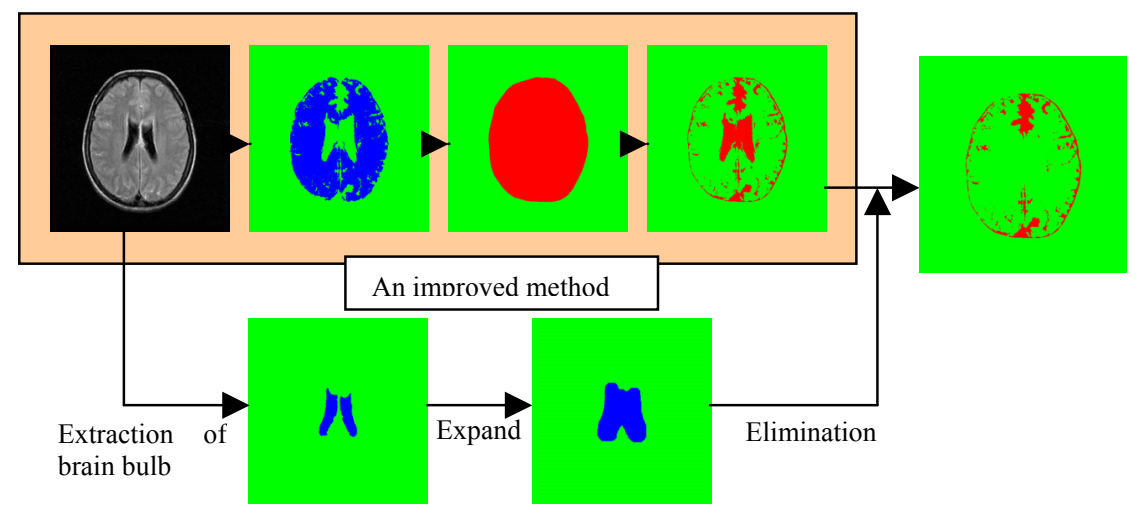

Figure 5: $\quad$ Elimination of area with high-intensity values.

\section{Evaluation}

In order evaluate our diagnosis support system, we had a questionnaire from three neuro surgeons. The neuro surgeons see both the original image and the estimated bleeding area image, and answer the following questionnaires. We show the eight images, and the neuro surgeons answer the questionnaire. Table 1 shows the result. Fig. 6 shows the final results. The left images are generated by our proposed method without improvements, and they show the wrong area (enclosed by green lines. The right images show the results of our improvement.

1. The preciseness of the extraction

1. There are many wrong extractions.

2. There are many right extractions, but a little wrong area (many extractions failure).

3. There are many right extractions with no failure.

4. There are many right extractions, but a little wrong area (too small extraction failure) .

5. There are many areas that fail to extract a bleeding area. 
2. The effectiveness of our diagnosis support system

1. It is useful for a diagnosis support system efficiently.

2. It is useful for a diagnosis support system.

3. Unknown

4. It is insufficient for a diagnosis support system.

5. It is not used for a diagnosis support system.

Table 1: $\quad$ A result of questionnaire.

\begin{tabular}{|l|l|l|}
\hline Questionnaire Item & Num & Ratio \\
\hline 1. 1. Many wrong extractions & 1 & 4.0 \\
\hline $\begin{array}{l}\text { 1. 2. Many right extractions, but a little wrong area (many } \\
\text { extractions failure). }\end{array}$ & 8 & 36.0 \\
\hline 1. 3. Many right extractions with no failure. & 13 & 52.0 \\
\hline $\begin{array}{l}\text { 1. 4. Many right extractions, but a little wrong area (too } \\
\text { small extraction failure) }\end{array}$ & 2 & 8.0 \\
\hline 1. 5. Many area to fail to extract bleeding areas & 0 & 0.0 \\
\hline & & \\
\hline 2.1. Useful as a diagnosis support system efficiently. & 3 & 12.5 \\
\hline 2. 2. Useful as a diagnosis support system & 18 & 75.0 \\
\hline 2. 3. Unknown & 0 & 0.0 \\
\hline 2. 4. Insufficient as a diagnosis support system & 3 & 12.5 \\
\hline 2. 5. Not useful as a diagnosis support system & 0 & 0.0 \\
\hline
\end{tabular}

\section{Conclusion}

In this paper, we proposed an improved diagnosis method for stroke, which estimates the risk-factor of stroke automatically from two-dimensional MR/FLAIR sequence images. By checking the gap of the brain, we found that the histogram of stroke has a similar pattern, and it can be used for diagnosis of stroke. In order to evaluate our approach, we had questionnaire from neuro surgeons, and we found that our approach is effective for diagnosis support of stroke.

We have two improvements for our proposed method. At first, we specify a threshold C automatically. Secondly, we also extract the brain bulb, and expand the area, and eliminate the bleeding area near the brain bulb. The two algorithms improve the score the questionnaire from three neuro surgeons.

The gap area is extracted by region growing method. However, most region growing methods are sensitive to the selection of the initial growing points (seeds), and the merging criteria parameters. In order to diagnosis automatically, we need to define a set of theoretical criteria, and a method that are insensitive to the selection of the initial growing points, such as Symmetric Region Growing [8]. We also need many clinical examples for improving the preciseness of diagnosis support. 

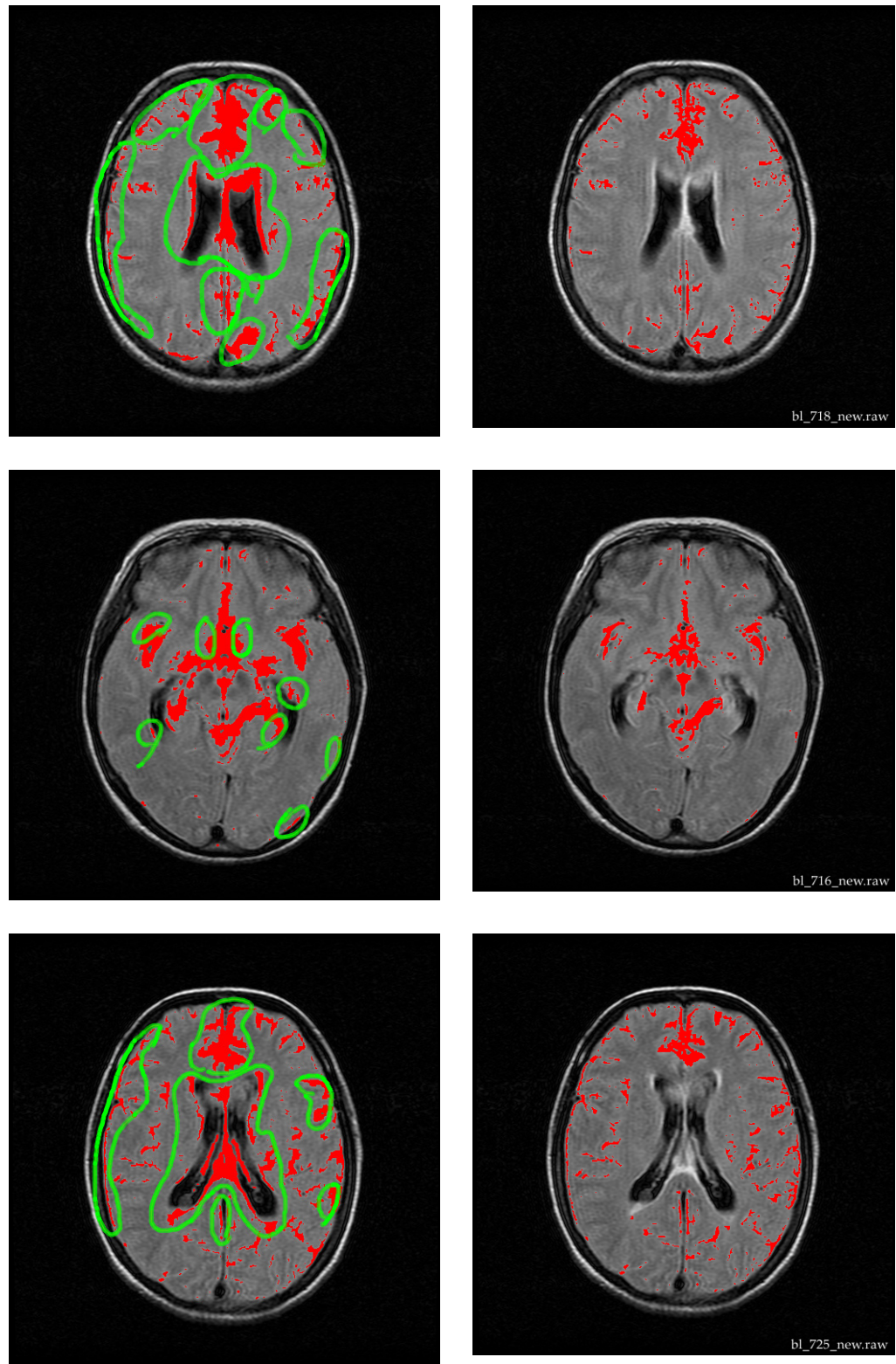

Figure 6: Final results (left images show the wrong area (enclosed by green lines), and the right image shows the improvement of our approach.

\section{References}

[1] H. Jiang, J. Toriwaki, and H. Suzuki, "Comparative performance evaluation of segmentation methods based on region growing and division", Syst. Comput. Jpn, Vol. 24, No. 13, pp. 28-42, 1993. 
[2] R. Adams and L. Bischof, "Seeded region growing”, IEEE Trans. Pattern Anal. Machine Intell, Vol. 16, No. 6, pp. 641-647, 1994.

[3] S. C. Zhu and A. Yuille, "Region competition: Unifying snakes, region growing, and Bayer/MDL for multiband image segmentation", IEEE Trans. Pattern Anal. Machine Intell, Vol. 18, No. 9, pp. 884-900, 1996.

[4] S. A. Hojiato Leslami and J. Kittler, "Region growing: A new approach", IEEE Trans. Image Processing, Vol. 7, pp. 1079-1084, 1998.

[5] M. Kass, A. Witkin, and D. Terzopoulos, "Snakes: Active Contour Models", International Journal of Computer Vision, 1, 4, pp. 321-331, 1988.

[6] L. D. Cohen, "On Active Contour Models and Balloons", CVGIP: Image Understanding, Vol. 53, No. 2, pp. 211-218, 1991.

[7] K. Sakaue and K. Yamamoto, "Active Net Model and Its Application to Region Extraction", The Journal of the Institute of Television Engineering of Japan, 45, 10, pp. 1155-1163, 1991.

[8] Shu-Yen and W. E. Higgins, "Symmetric Region Growing", IEEE Trans. Image Processing, Vol. 12, No. 9, pp. 1007-1015, 2003. 